\title{
A Greener Way to Screen Toothpaste for Diethylene Glycol
}

\author{
Yale Fu, Zhigang Hao, Barry Parker, Michael Knapp \\ ${ }^{1}$ Global Analytical Science Department, Colgate-Palmolive Company, Piscataway, USA \\ E-mail: zhigang.hao@gmail.com \\ Received October 14, 2011; revised November 21, 2011; accepted December 3, 2011
}

\begin{abstract}
A method developed for the screening of diethylene glycol (DEG) in toothpaste was released by the FDA in 2007. This method could not only quantify the DEG but also confirm if any potential interfering peak is present. However, disadvantages of this method such as intermittent shortages of the key reagent acetonitrile and the shorter than expected column-life issues have prompted a search for alternative solutions. An improvement with an alternate "greener" extraction solvent is presented, and the method comparison and validation are described in this article. The greener extraction solvent, ethanol with limited water, provided a better efficiency for the toothpaste sampling procedures. The limit of detection (LOD) and limit of quantitation (LOQ) are $0.0025 \%$ and $0.0084 \%$ in (w/w) unit, respectively. The sample recovery is $101.2 \%$.
\end{abstract}

Keywords: Greener Way, Diethylene Glycol, DEG, Ethanol, Gas Chromatography-Mass Spectrometry, Carryover

\section{Introduction}

Diethylene glycol (DEG, CAS number 111-46-6) is a clear, colorless, practically odorless, viscous, and hydroscopic liquid with a sweet taste. It is miscible in water, alcohol, and acetonitrile. In addition to its use in a wide range of industrial products including dehydration of natural gas, production of polyurethanes and unsaturated polyester, antifreeze and brake fluids [1,2], DEG can be formed by polymer degradation in biomaterials [3] and other sources [4]. A number of DEG detection methods have been developed over the years. Some methods focus on raw materials such as glycerin [5], ethylene, and propylene [6-8]. Other methods are devoted to DEG detection in finished products such as cosmetics [9], oral care products [10-14], food [15] and pharmaceuticals [16]. DEG detection methods in biological studies [15], environmental polution samples [17] and phytoremediation [18] have also been reported. A common characteristic of most DEG detection methods is that the sample matrix must be predictable and the peak identity is not a critical issue because matrix interference can be avoided and removed by separation efforts. Method specific applications have been validated with a known individual matrix. Difficulties arise when the product matrix is unknown. As a result, the potential for peak interference presents a significant challenge to method specificity.

The Food and Drug Administration (FDA) endorsed a method for DEG detection in toothpaste [19]. This method provides a resolute ability to confirm the presence of DEG in a toothpaste matrix, which could not only quantify the DEG contents but also identify if any interfering peaks are present. The method employs the use of gas chromatography (GC) coupled to a mass spectroscopy detector (MS). The GC provides the chromatographic separation of the individual volatile components which are then sent into the full scan MS for detection. The unique ability of the MS detector allows each individual component to be identified and can distinguish if any interfering peaks are present. The method has helped the Colgate-Palmolive Company successfully screen toothpaste products and provide brand protection globally. However, several issues have been identified with the method. Firstly, the solvents (acetonitrile and water) used in the sampling procedure are not "friendly" with respect to either the environment or the GC column. Secondly and more importantly, the issues with DEG carryover and its retention time shift become more and more pronounced after numerous samples have been run on the GC instruments. Finally, the acetonitrile shortage between 2008 and 2010 significantly increased the method operating cost to an unacceptable level. As part 
of a new solvent strategy, it was decided to search for a greener alternative to acetonitrile and without sacrificing performance. Not only was a greener and more cost effective solvent (ethanol) identified, but also a more column friendly sample preparation. This new method has been validated on dentifrice products for DEG and is currently being used at Colgate-Palmolive Company.

\section{Experimental}

\subsection{Materials and Reagents}

Diethylene glycol (99\%), anhydrous granule sodium sulfate $\left(\mathrm{Na}_{2} \mathrm{SO}_{4}\right)$ and $710-1180$ microns of glass beads were purchased from Sigma-Aldrich (St. Louis, MO, USA). 1,3-propanediol (99\%) was from Alfa Aesar (Ward Hill, MA, USA). 15-mL polypropylene centrifuge tubes were obtained from VWR (West Chester, PA, USA). ASTM type 1 water (at point of delivery) was prepared internally from ELGA PURELAB prima 7/ Purelab Ultra Analytic (Lowell, MA, USA), acetonitrile (HPLC grade) was purchased from JT Baker (Philipsburg, NJ, USA) and anhydrous ethyl alcohol (ACS/USP grade) was purchased from PHARMCO-AAPER (Brookfield, CT, USA). The anhydrous ethyl ethanol needs to be kept in seal until use.

\subsection{Instrumentation}

The Eppendorf centrifuge 5810R was used to centrifuge the toothpaste sample for $10 \mathrm{~min}$ at $5000 \mathrm{~g}$ before the GC-MS analysis. A Genie 2 vortex mixer was used to assist sample dispersion in a minimum of time.

The gas chromatography system 5890 with 5972 MS detector plus 7673 autosampler-split/splitless injector or the gas chromatography system 6890 with 5973 MS selective detector plus 6890 autosampler-split/splitless injector (GC-EI-MS, Agilent Technologies, Santa, Clara, CA, USA) was used for DEG analysis. Separation was accomplished using a $30 \mathrm{~m}$ long Stabilwax capillary column, $0.25 \mathrm{~mm}$ internal diameter (I.D.) and $0.25 \mu \mathrm{m}$ film thickness (Restek, Bellefonte, PA, USA). The $1 \mu \mathrm{L}$ sample was injected with the split mode at ratio of 1:20. The oven temperature was initially held at $100^{\circ} \mathrm{C}$ for 1 minute. Thereafter the temperature was raised at $10^{\circ} \mathrm{C}$ $/ \min$ until $250^{\circ} \mathrm{C}$ and held for 4 minutes. Helium was used as the carrier gas and delivered at a constant flow rate at $1 \mathrm{~mL} / \mathrm{min}$ (the pressure at $8.2 \mathrm{psi}$ and velocity at $37 \mathrm{~cm} / \mathrm{sec}$ ). The injector temperature was set at $250^{\circ} \mathrm{C}$ and the interface temperature was $250^{\circ} \mathrm{C}$. The MS detectors were tuned with the standard spectrum autotune, and the MS data (total ion chromatogram, TIC) were acquired with either the full scan mode (m/z of $29-400$ at a scan rate of $4 \mathrm{scan} / \mathrm{sec}$ or selected ion monitoring (SIM) using the electron ionization (EI) mode for the fragments at 31,45 and $75 \mathrm{~m} / \mathrm{z}$ with an electron energy of $70 \mathrm{eV}$. The MS source temperature is $230^{\circ} \mathrm{C}$ and quat temperature is $150^{\circ} \mathrm{C}$. The retention time of DEG is about $9.9 \mathrm{~min}$, and the solvent delay is 4 minutes.

\subsection{Blank, Standard and Internal Standard Solutions}

A blank solution which consists of $10 \mathrm{~mL}$ of extraction solvent (either $50 \%$ acetonitrile-water or $98 \%$ ethanolwater) taken through the entire procedure including the addition of the internal standard was evaluated to make sure that there was no contamination from the reagents and containers.

Approximately 1.0 gram of DEG standard was weighed into a $100 \mathrm{~mL}$ volumetric flask and dissolved with a $50 \%$ aqueous acetonitrile or a $98 \%$ aqueous ethanol solvent, respectively. A series of standard solutions with a 1:3 dilution from this standard stock solution were made for method evaluation.

Approxiamtely 0.5 gram of internal standard, 1,3propanediol, was weighed into a $100 \mathrm{~mL}$ volumetric flask and dissolved with a $50 \%$ aqueous acetonitrile or a $98 \%$ aqueous ethanol solvent, respectively. $0.1 \mathrm{~mL}$ of this solution was added into $1 \mathrm{~mL}$ of each sample extract or standard solutions in the autosampler vial prior to GC-MS analysis. The fixed concentration is $0.05 \%$. The internal standard with $50 \%$ aqueous acetonitrile solvent was for the original method, and the internal standard with a $98 \%$ aqueous ethanol solvent was for the new developed method.

\subsection{QC and Spiking Sample Preparation}

The QC samples were set up at $0.5 \mathrm{mg} / \mathrm{mL}$ and 0.1 $\mathrm{mg} / \mathrm{mL}$ levels. The QC sample at $0.5 \mathrm{mg} / \mathrm{mL}$ of DEG was analyzed at the beginning and the end of the sample set to provide a basis for quantitative evaluation and to monitor the amount of drift during the analysis of the set of samples. QC sample at $0.1 \mathrm{mg} / \mathrm{mL}$ of DEG is analyzed at the center of the sample set for the threshold level DEG detection.

The standard spiking solution was made from the standard stock solution with a $1: 2$ dilution, and $0.2 \mathrm{~mL}$ of this standard spiking solution was directly added to the representive uncontaminated sample before proceeding with the sample preparation procedures.

\subsection{Sample Preparation}

For comparison purposes, both the original and new 
sampling procedures are described here.

\subsubsection{Sampling Preparation for the New Developed Method}

Approximately 1.0 gram of toothpaste sample was weighed into a $15 \mathrm{~mL}$ polypropylene centrifuge tube. To wet the toothpaste materials, $0.2 \mathrm{~mL}$ of water was added. To assist toothpaste sample suspension, 0.5 gram of glass beads (710 - 1180 micros, Sigma-Aldrich , St. Louis, MO, USA) were premixed well by using a Genie 2 vortex mixer (Scientific Industrial, Inc., Bohemia, NY, USA). After vortexing, $9.8 \mathrm{~mL}$ of ethanol and 2 grams of anhydrous granule sodium sulfate was added; after throrough mixing via vortex, the centrifuge was used for 10 minutes at $5000 \mathrm{~g}$ to isolate the supernatant. The supernatant was filtrated by a syringeless filter device with a $0.45 \mu \mathrm{m}$ PVDF membrance from Whatman (Clifton, NJ, USA) for GC-MS analysis.

\subsubsection{Sampling Preparation for the Original Method}

Approximately 1.0 gram of toothpaste was weighed into a $15-\mathrm{mL}$ polypropylene centrifuge tube from VWR (West Chester, PA, USA) and $5 \mathrm{~mL}$ of water was added. Mix well to thoroughly disperse the entire sample. A Genie 2 vortex mixer (Scientific Industrial, Inc., Bohemia, NY, USA) was used to assist this process. After the toothpaste sample was fully suspended, $5 \mathrm{~mL}$ of acetonetrile in two portions with thorough mixing between each addition was added. Centrifuge for 10 minutes at $5000 \mathrm{~g}$. The supernatant was filtrated by a syringeless filter device with a $0.45 \mu \mathrm{m}$ PVDF membrance from Whatman (Clifton, NJ, USA) for GC-MS analysis.

\section{Results and Discussion}

\subsection{The Specificity from the Original GC-MS Method}

An important component in toothpaste products is the flavor, which can be composed of many different volatile ingredients and produce potential interference to DEG during the GC separation. For the known toothpaste samples, all potential interference can be avoided with GC separation efforts during the method development because the matrix flavors are known. However, for unknown toothpaste samples, the potential interference is not predictable because the matrix flavors are not known. Two extreme examples are presented in Figure 1 and the interfering peaks can be located just before (Figure 1(a)) and after (Figure 1(b)) the DEG peak.

MS full scan mode is necessary to confirm if the unknown samples contain DEG. In the practical application, single ion monitoring (SIM) mode of MS detection can

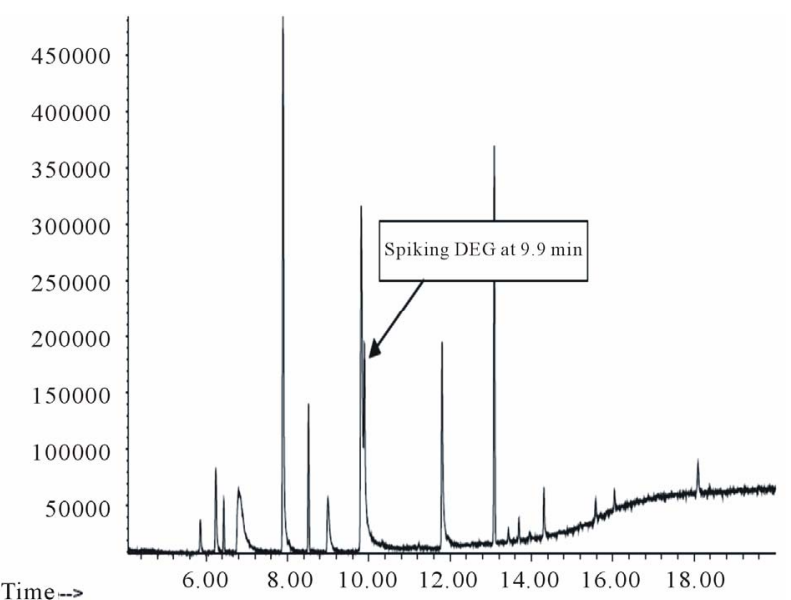

(a)

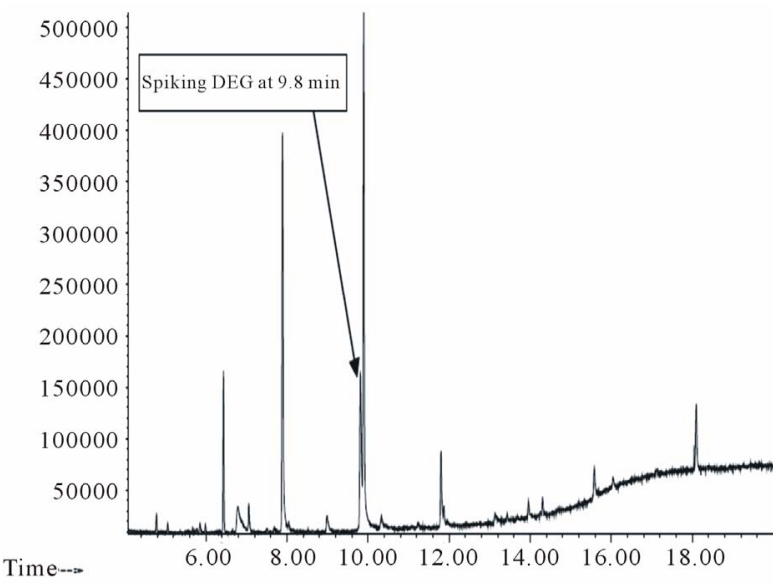

(b)

Figure 1. Two typical GC-MS chromatograms of unknown toothpaste spiked with DEG analytes. Figure 1(a) (top) presents an interfering peak just before the DEG peak at retention time $9.9 \mathrm{~min}$ and Figure 1(b) (bottom) presents an interfering peak just after the DEG peak at retention time $9.8 \mathrm{~min}$. (Note that DEG peak retention time was shifted after numerous sample analyses).

also be used to screen for DEG in the toothpaste. The SIM mode can provide better sensitivity, but the peak identity is not reliable even when the ratio of the individual fragment from SIM mode is monitored. Figure 2(a) exhibits a MS spectrum with a full scan mode detection of the DEG standard, which provided $90 \%$ match quality to the NIST 2002 database. Figure 2(b) displays a MS spectrum with a SIM mode detection and the individual fragment ratios at $\mathrm{m} / \mathrm{z} 31,45$, and 75 are right when the DEG concentration is above the LOQ level of full scan detection. When DEG cencetration reached the LOQ levels in the full scan mode, the ratios at $\mathrm{m} / \mathrm{z}$ of 31 , 45 , and 75 started to be twisted, which is presented in Figure 2(c). 


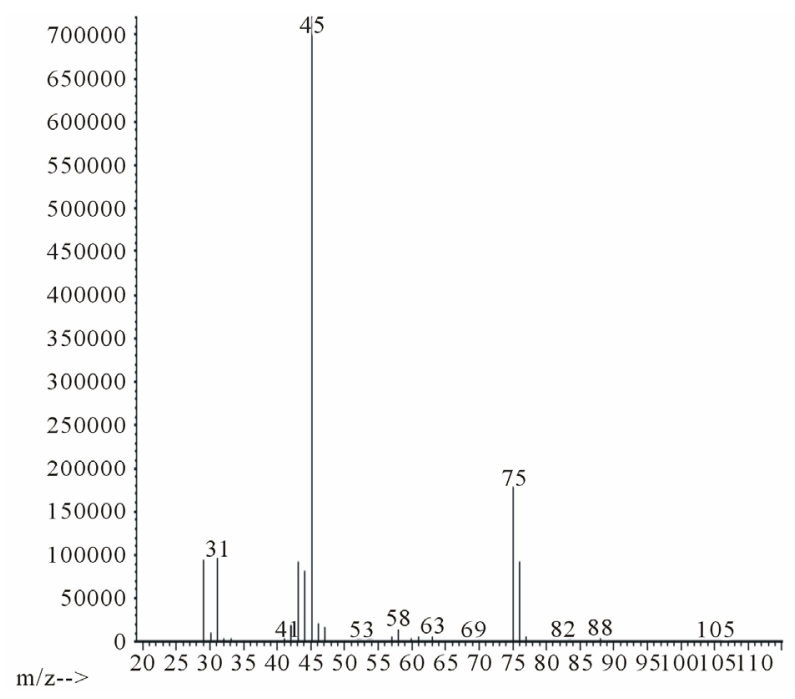

(a)

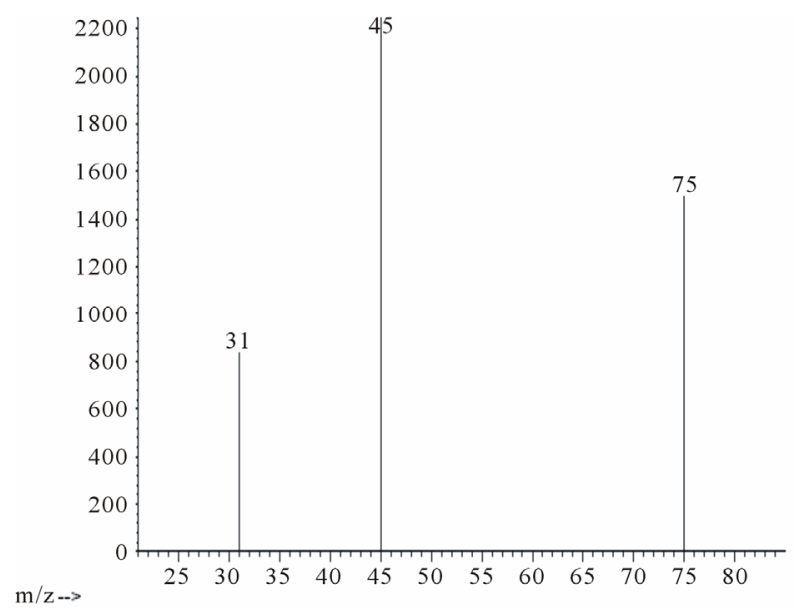

(b)

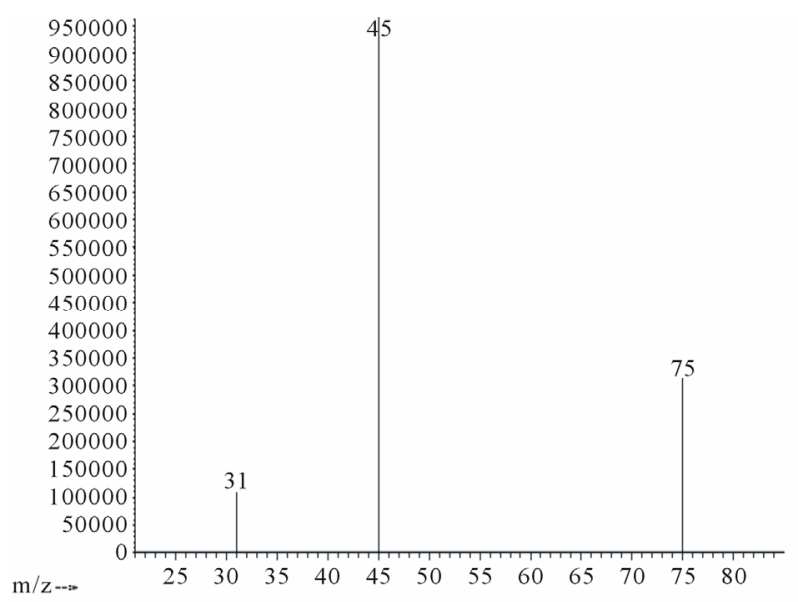

(c)

Figure 2. DEG mass spectra obtained from full scan and single ion monitroing (SIM) modes. 2(a) (top) was from full scan detection; 2(b) (middle); and 2(c) (bottom) were from SIM detections.

\subsection{The Challenges of the Original GC-MS Method}

Toothpaste materials cannot be dissolved or suspended well in organic solvents such as polar methanol used during the sampling procedure. Water is usually a good media for toothpaste sampling. However, water is a less-than-ideal solvent from a GC point of view. The problems associated with water include a large vapor expansion volume and perceived chemical damage to the stationary phase [20]. Based on the solvent expansion calculator software provided by Agilent for GC instrument, the approximate vapor volume of $50 \%$ acetonetrile-water solvent under the flow rate of $1 \mathrm{~mL} / \mathrm{min}$ (pressure approximately $8.2 \mathrm{psi}$ ) can be larger than $1000 \mu \mathrm{L}$, which surpasses the volume of all commercially avaiable GC injection liners. Therefore, the DEG carryover was observed after two blank injection intervals during subsequent sample or standard injections. Carryover is defined as the appearance of a compound in a blank sample, especially when the blank sample is injected immediately after an injection of a sample or standard containing high concentration of analytes, which can be a result of the backflash phenomenon [20]. In addition, the boiling point of DEG is $244^{\circ} \mathrm{C}-245^{\circ} \mathrm{C}$ which requires a terminal temperature of $250^{\circ} \mathrm{C}$ in the GC oven program. Serious column bleeding can be observed in Figure 1 due to the high ratio of water in the injected solvent. DEG retention time continuously dropped after a larger number of sample injections. To improve the original FDA method [19], a new sampling procedure was developed in this study.

The water volume in the revised sampling procedures has been limited to $200 \mu \mathrm{L}$ ( $2 \%$ of total sampling solvent). Water is required to wet the toothpase matrix, and many toothpaste samples cannot be suspended without using the water. To improve the sampling solubility and suspension, a solvent with a stronger hydrogen-bonding capability, ethanol, was utilized to replace the acetonetrile. Ethanol is a cheaper and more environmentallyfriendly solvent compared to acetonitrile. To enhance toothpaste sample suspension, glass beads (710 - 1180 microns size) and anhydrous granule sodium sulfate were applied. Anhydrous sodium sulfate provides two critical functions, it mechanically enhances the toothpaste suspension, and it removes the water from the ethanol phase [21]. All the tested toothpaste samples showed good suspension with the assistance of a Genie 2 vortex mixer (Scientific Industrial, Inc., Bohemia, NY, USA).

\subsection{Method Evaluation}

To evaluate the solvent replacement, several method validation parameters have been measured and compared 
between the extraction solvents of $50 \%$ aqueous acetonetrile and $98 \%$ aqueous ethanol. A typical chromatogram of DEG with the improved ethanol extraction procedure is shown in Figure 3. All the data were obtained from the newer instrument, the gas chromatography system 6890 with 5973 MS selective detector plus 6890 autosmapler-split/splitless injector.

\subsubsection{Linearity of Response, Limits of Detection (LOD) and Quantitation (LOQ)}

The calibration responses were obtained by plotting the peak area ratio between the DEG standard concentration range of $0.16 \%$ down to $0.01 \%$ and 1,3 -propanediol at a fixed concentration of $0.05 \%$. As shown in Table 1 , good linearities were obtained for both $98 \%$ aqueous ethanol and $50 \%$ aqueous acetonitrile.

The LODs and LOQs by GC-MS in the full scan mode are shown in Table 1. The LOD and LOQ with 50\% aqueous acetonitrile extraction are $0.0044 \%$ and $0.0146 \%$, respectively. The better LOD and LOQ with $98 \%$ aqueous ethanol extraction are $0.0025 \%$ and $0.0084 \%$. The sensitivity of the LOD and LOQ can be significantly improved with the SIM mode detection, but this is not suitable for screening unknown toothpaste samples for DEG.

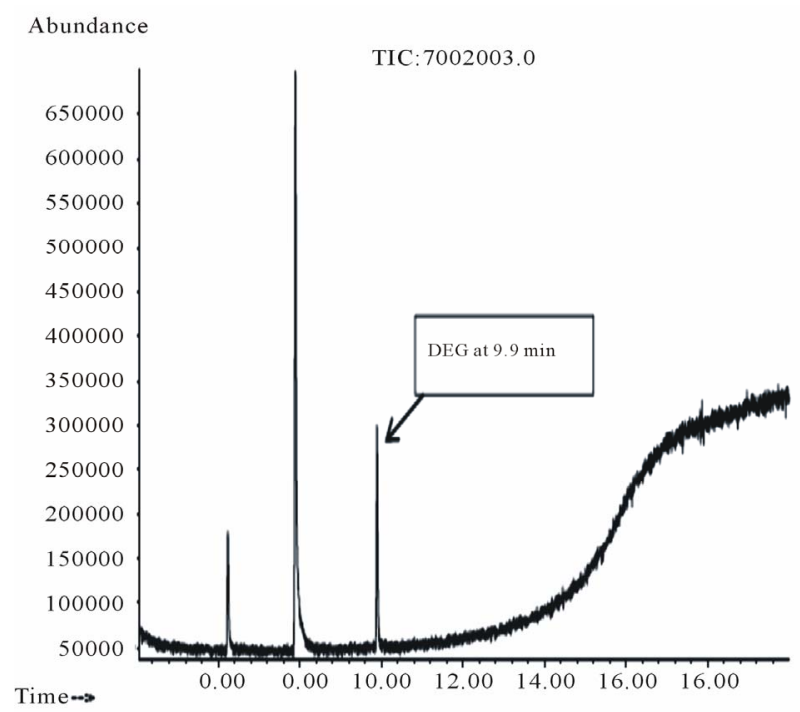

Figure 3. A typical GC-MS chromatogram of DEG with the improved ethanol extraction procedure.

Table 1. Calibration parameters and sample recovery.

\begin{tabular}{cccccc}
\hline $\begin{array}{c}\text { Sampling } \\
\text { solvents }\end{array}$ & $\mathrm{R}^{2}$ & LOD & LOQ & $\begin{array}{c}\text { RSD } \\
\%\end{array}$ & $\begin{array}{c}\text { Recovery } \\
\%\end{array}$ \\
\hline $\begin{array}{c}50 \% \text { aqueous } \\
\text { ACN }\end{array}$ & 0.9999 & $0.0044 \%$ & $0.0146 \%$ & $12.4 \%$ & $87.5 \%$ \\
$\begin{array}{c}98 \% \text { aqueous } \\
\text { EtOH }\end{array}$ & 1.0000 & $0.0025 \%$ & $0.0084 \%$ & $6.7 \%$ & $101.2 \%$ \\
\hline
\end{tabular}

\subsubsection{Method Accuracy and Precision}

A toothpaste without fluoride or DEG (Grins \& Giggles, by Gerber Product Company, Fremont, MI, USA) was used as the spiking matrix to perform the analyte recovery for accuracy evaluation. The recovery values are the results from five injections of spiked standards in matrix following the entire procedure from sample suspension, centrifuging and GC-MS analysis.

The precision, as given by relative error ( $\mathrm{RE} \%)$ and relative standard deviation ( $\mathrm{RSD} \%$ ), respectively, was evaluated (Table 1) by analyzing five replicates of DEG standard at $0.01 \%$.

\subsubsection{Carryover}

To evaluate the carryover effect, a blank sample was set next to the upper limit of quantification control sample (QC sample at $0.5 \mathrm{mg} / \mathrm{mL}$ ). A significant carryover peak of DEG was observed with $50 \%$ aqueous acetonitrile extraction procedure but no peak was present after the extraction solvent was replaced by $98 \%$ aqueous ethanol solvent.

\section{Conclusions}

When the sampling solvent was switched from $50 \%$ acetonitrile-water to $98 \%$ ethanol-water, all the validation data from carryover, linearity, sensitivity, precision to accuracy exhibited a positive improvement. Ethanol is not only more cost effective than acetonitrile but also more friendly (greener) to the environment. Moreover, column life with this newly developed method is longer than with the original method.

\section{Acknowledgements}

Some experimental procedures and experimental conditions are copied from the original FDA GC-MS method, and we would like to thank its authors, Jonathan Lizau and Kevin Mulligan.

\section{References}

[1] R. J. Lewis and S. Hawley, "Condensed Chemical Dictionary," Van Nostrand Reinhold Company, New York, 1993.

[2] J. M. Marraffa, M. G. Holland, C. M. Stork, C. D. Hoy and M. J. Hodgman, "Diethylene Glycol: Widely Used Solvent Present Serious Poisoning Potential," The Journal of Emergency Medicine, Vol. 35, No. 4, 2008, pp. 401-406. doi:10.1016/j.jemermed.2007.06.025

[3] A. Ghaffara, P. G. Verschuren, J. A. J. Geenevasen, T. Handels, J. Berard, B. Plum, A. A. Dias, P. J. Schoenmakers and $\mathrm{Sj}$. van der Wal, "Fast in vitro Hydrolytic Degradation of Polyester Urethane Acrylate Biomaterials: 
Structure Elucidation, Separation and Quantification of Degradation Products," Journal of Chromatography A, Vol. 1218, No. 3, 2011, pp. 449-458. doi:10.1016/j.chroma.2010.11.053

[4] G. Stefania, B. Maura, V. L. Claudia, P. Barbara, M. Ginevra and R. Francesco, "Biological Effects of Diethylene Glycol (DEG) and Produced Waters (PWs) Released from Offshore Activities: A Multi-Biomarker Approach with the Sea Bass Dicentrarchus labrax," Environmental Pollution, Vol. 157, No. 11, 2009, pp. 3166-3173. doi:10.1016/j.envpol.2009.05.021

[5] C. M. Gryniewicz, J. A. Spencer, M. Hankins and J. F. Kauffman, "Spectroscopic Methods for Rapid Determination of Diethylene Glycol in Glycerin," American Pharmaceutical Review, Vol. 10, 2007, pp. 1-4.

[6] G. Holloway, R. Maheswaran, A. Leeks, S. Bradby and S. Wahab, "Screening Method for Ethylene Glycol and Diethylene Glycol in Glycerin-Containing Products," Journal of Pharmaceutical and Biomedical Analysis, Vol. 51, No. 3, 2010, pp. 507-511.

doi:10.1016/j.jpba.2009.08.025

[7] X. Li, S. Arzhantsev, J. F. Kauffman and J. A. Spencer, "Detection of Diethylene Glycol Adulteration in Propylene Glycol-Method Validation through a Multi-Instrument Collaborative Study," Journal of Pharmaceutical and Biomedical Analysis, Vol. 54, No. 5, 2011, pp. 1001- 1006. doi:10.1016/i.jpba.2010.11.042

[8] ASTM E611-08 in ASTM, "American Society for Testing and Matericals, Standard Test Methods for Low Concentrations of Diethlyene Glycol in Ethylene Glycol by Gas Chromatography," Annual book of ASTM standard, Philadelphia.

[9] K. Molever, "Simplfied Assay of Diethylene Glycol and Ethylene Glycol in Various Raw Materials by Capillary Gas Chromatography," Society of Cosmetic Chemists, Vol. 61, 2010, pp. 225-234.

[10] A. Kenyon, X. Shi, Y. Wang, W. H. Ng, "Simple on-Site Detection of Diethylene Glycol/Ethylene Glycol Contamination of Glycerin and Glycerin-Based Raw Materials by Thin-Layer Chromatography," Journal of AOAC International, Vol. 81, 1998, pp. 44-50.

[11] J. Ding, H. Gu, S. Yang, M. Li, J. Li and H. Chen, "Selective Detection of Diethylene Glycol in Toothpaste Prod- ucts Using Neutral Desorption Reactive Extractive Electros- pray Ionization Tandem Mass Spectrometry," Analytical Chemistry, Vol. 81, No. 20, 2009, pp. 86328638. doi:10.1021/ac9013594

[12] F. Hernandez, M. Ibanez and J. V. Sancho, "Fast Deter- mination of Toxic Diethylene Glycol in Toothpaste by Ultra-Performance Liquid Chromatography-Time of Flight Mass Spectrometry," Analytical and Bioanalytical Chemistry, Vol. 391, No. 3, 2008, pp. 1021-1027. doi:10.1007/s00216-008-2082-0

[13] M. Lopez-Sanchez, A. Dominguez-Vidal, M. J. AyoraCanada and A. Molina-Diaz, "Assessment of Dentifrice Adulteration with Diethylene Glycol by Means of ATRFTIR Spectroscopy and Chemometrics," Analytica Chimica Acta, Vol. 620, No. 1-2, 2008, pp. 113-119. doi:10.1016/j.aca.2008.05.032

[14] X. Xing, X. Shi, H. Zhang, W. Wang and J. Ye, "Determination of Diethylene Glycol in Toothpaste by Capillary Electrophoresis with Electrochemical Detection," Microchimica Acta, Vol. 167, 2009, pp. 297-302.

[15] A. A. Rahim, B. Saad, H. Osman, N. Hashim, S. Yahya and K. M. Talib, "Simultaneous Determination of Diethylene Glycol, Diethylene Glycol Monoethyl Ether, Coumarin and Caffeine in Food Items by Gas Chromatography," Food Chemistry, Vol. 126, No. 3, 2011, pp. 1412-1416. doi:10.1016/j.foodchem.2010.11.121

[16] T. Zhou, H. Zhang and G. Duan, "Simultaneous Determination of Diethylene Glycol and Propylene Glycol in Pharmaceutical Products by HPLC after Precolumn Derivatization with P-Toluenesulfonyl Isocyanate," Journal of Separation Science, Vol. 30, No. 16, 2007, pp. 2620 2627. doi: $10.1002 /$ jssc. 200700097

[17] A. Cappiello, G. Famiglinia, P. Palma, V. Termopolia, H. Trufelli, R. Di Mentob and M. Mannozzi, "LC-ESI-MS Determination of Diethylene Glycol Pollution in Sea Water Samples Collected around Gas Extraction Platform Plants," Talanta, Vol. 80, No. 1, 2009, pp. 257-262. doi:10.1016/j.talanta.2009.06.067

[18] P. Teamkao and P. Thiravetyan. "Phytoremediation of Ethylene Glycol and Its Derivatives by the Burhead Plant (Echinodorus cordifolius (L.): Effect of Molecular Size," Chemosphere, Vol. 81, No. 9, 2010, pp. 1069-1074. doi:10.1016/j.chemosphere.2010.09.049

[19] http://www.fda.gov/Food/ScienceResearch/LaboratoryM ethods/DrugChemicalResiduesMethodology/ucm113209. htm

[20] E. Kuhn, "Water Injections in GC-How Wet Can You Get?” LCGC North America, Vol. 20, 2002, pp. 474-478.

[21] F. J. Schenck, P. Callery, P. M. Gannett, J. R. Daft and S. J. Lehotay, "Comparison of Magnesium Sulfate and Sodium Sulfate for Removal of Water from Pesticide Extracts of Foods," Journal of AOAC International, Vol. 85, 2002, pp. 1177-1180. 